\title{
Closing up onto our sun: solar imaging
}

\author{
Kushagra Shrivastava ${ }^{1}$, Keith Wen Kai Chia ${ }^{2}$, Kang Jun Wong $^{1}$, Alfred Yong Liang Tan ${ }^{1}$ \\ and Hwee Tiang Ning ${ }^{1}$ \\ ${ }^{1}$ National Junior College, 37 Hillcrest Road, Singapore \\ ${ }^{2}$ School of Science and Technology, 1 Technology Drive, Singapore
}

\begin{abstract}
Solar activity research provides insight into the Sun's past, future (Science Daily, 2018). The solar activity includes observations of large numbers of intense sunspots, flares, and other phenomena; and demands a wide range of techniques and measurements on the observations. This research needs long term data collection before critical analyses can occur, to generate meaningful learning and knowledge. In this project, we will use solar imaging to make observations of solar activity, and take our baby steps to make contributions in citizen science. Observations will be made in 3 wavelengths to gain a more thorough analysis by looking at different perspectives of the Sun, namely H-Alpha, Calcium-K, and white light.
\end{abstract}

\section{Introduction}

In today's world, we rely heavily on satellites, radio communication and other such technology. They have made our life so much easier and convenient. Can you even imagine your life without YouTube, Instagram or Netflix? However, our closest star, the Sun, is a threat to this technology. Even though the Sun is the source of thermal energy on earth which makes it habitable for us, it is a double-edged sword. Solar activities like solar flares, coronal mass ejections and coronal holes bombard our satellites with charged particles that disrupt their normal functioning ${ }^{[1]}$. On March 13 1989, Quebec suffered a blackout as a result of a solar flare ${ }^{[2]}$. Due to such incidents, it is necessary for us to study about the Sun in order to predict when a solar activity might occur. Unfortunately, even after decades, we are not completely able to predict these events. Thus, everyone's observation is required - both amateur and professional solar astronomers - in order for us to predict when such events might take place.

\section{Aim}

The aim of our project is to document the activities on the Sun via optical means and analyze them in order to obtain more knowledge about the Sun and its activities.

\section{Methodology}

\subsection{Equipment used}

\subsubsection{Hardware}

- 2 Go2Nova telescope mount controllers (See Figure. 1)

*Email: kushagrashrivastava22@,gmail.com 


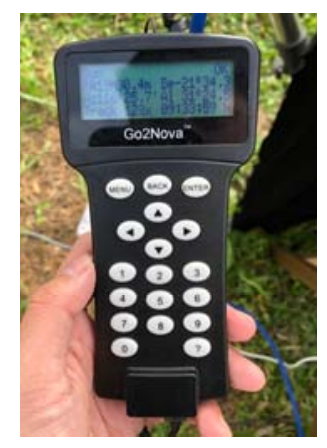

Figure 1. Go2Pro Controller

- CEM25P and CEM60 mount (See Figure 2)

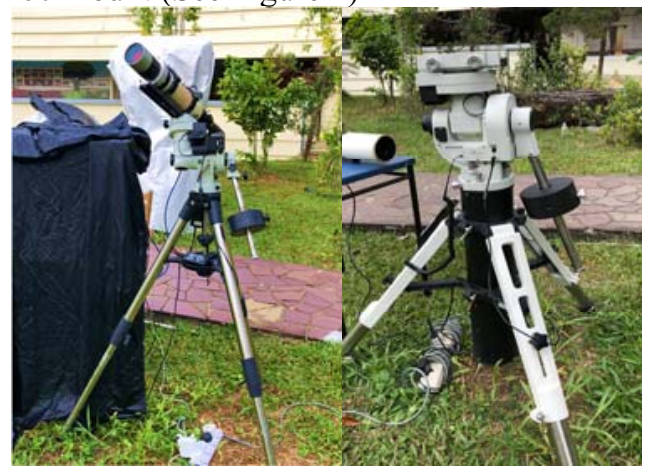

Figure 2. Borg $76 \mathrm{~mm}$ refractor mounted on CEM25P mount (left) and CEM60 mount (right)

- $\quad$ Borg $76 \mathrm{~mm}$ achromat refractor (See Figure 2 left)

- $\quad$ Megrez-90 refractor (See Figure 3)

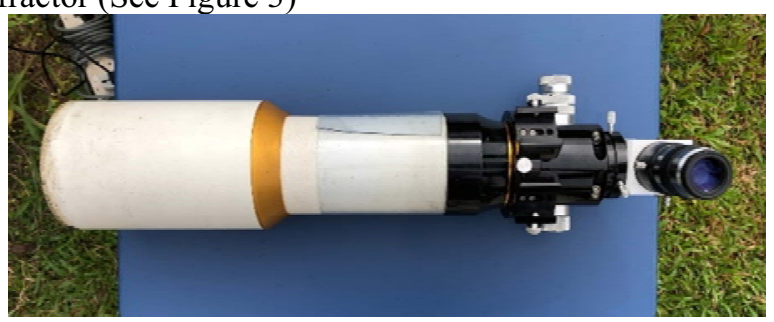

Figure 3. Megrez-90 Refractor

- White Light filter (See Figure 4)

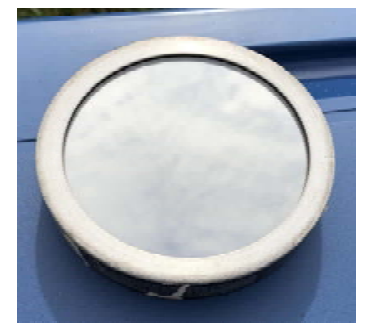

Figure 4. White Light Filter 
- Calcium-K filter (See Figure 5)

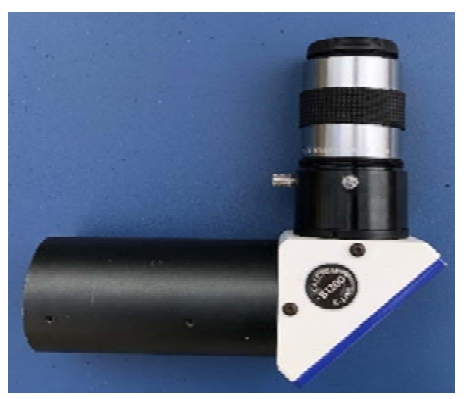

Figure 5. Calcium-K Filter

- Double stacked H-alpha Etalon (See Figure 6)

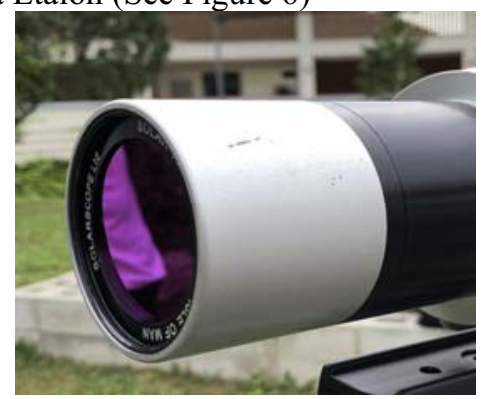

Figure 6. H-Alpha etalon

- $\quad$ ASI174MM Camera (See Figure 7)

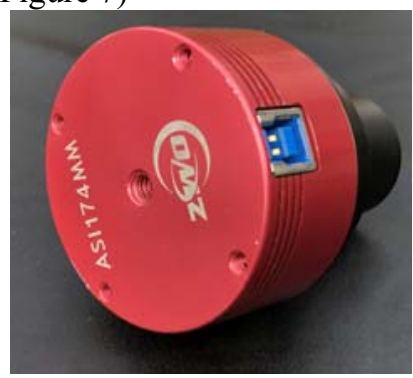

Figure 7. ASI174MM

\subsubsection{Software}

- FireCapture

- AutoStakkert3!

- ImPPG

- Adobe Photoshop

\subsection{Setting up mounts}

The mounts were setup using the following procedure:

i. Place the mount on a flat ground 
ii. Attach counterweight

iii. Perform polar alignment to the geographical North Pole

iv. Place telescope on the mount rails

v. Plug the Go2Nova controller onto the mount

\subsection{Data capturing}

For data capturing, the following procedure was used:

i. Using the Go2Nova controllers connected to the CEM25P and CEM60 separately, the telescope was aimed at the Sun.

ii. The camera, ASI174MM, was attached to a laptop with FireCapture.

iii. The mount controller was then used for fine adjustments and to bring the Sun in the center of the frame.

iv. Exposure was increased to see the three mirrored images of the Sun (See Figure 8), followed by decreasing it so that only the brightest one is visible.

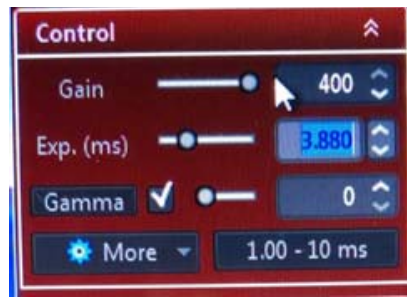

Figure 8. Increasing Exposure on FireCapture

v. The gain was increased or decreased as required.

vi. The video was captured with a time limit of 15 seconds.

\subsection{Data processing}

The problem with data processing is that there is no fixed set of parameters that can be used to process all images. It largely depends on the weather conditions and the quality of equipment used. In order to determine the best set of values for the various variables, we tried many and varied permutations of values, over many hours of processing. The values mentioned in the procedure below are the most used ones. However, it should be noted that every image requires slightly different parameters for fine-tuning. Thus, it is possible that the parameters mentioned afterward will not yield a good image for other users.

\subsubsection{AutoStakkert3!}

The recorded video was stacked using AutoStakkert3! First, the frames of the video were analyzed to obtain a frame quality curve of the video at surface image stabilization. Laplace transformation was on with noise-robust of 4 units. Then the frame quality curve was analyzed to see the percentage of frames with good quality followed by setting of alignment points at 16-unit size. $40-50 \%$ of the total frames were chosen for stacking. The sharpening box was unchecked and the video was stacked in TIF format. (See Figure 9) 


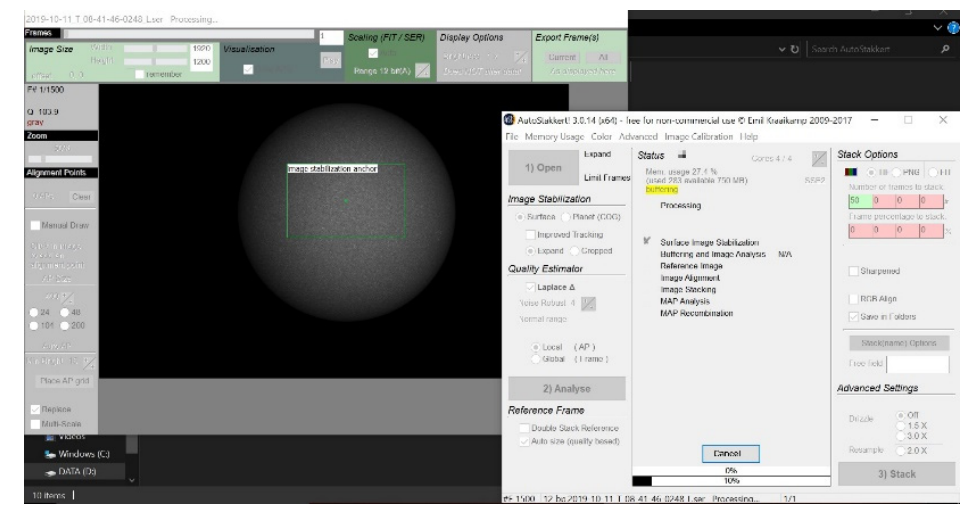

Figure 9. AutoStakkert3! analyzing a video

\subsection{2 $\operatorname{ImPPG}$}

The stacked image was sharpened in ImPPG. The tone curve of the image is stretched to fit the histogram in order to get a higher contrast (See Figure 10). Then, the Lucy-Richardson deconvolution method ${ }^{[6]}$ was used to sharpen the image. The values of sigma, amount and unsharp-masking were changed as per the image quality. Usually, the value of sigma and unsharp-masking was about the same. The final picture was then saved as a BMP file.

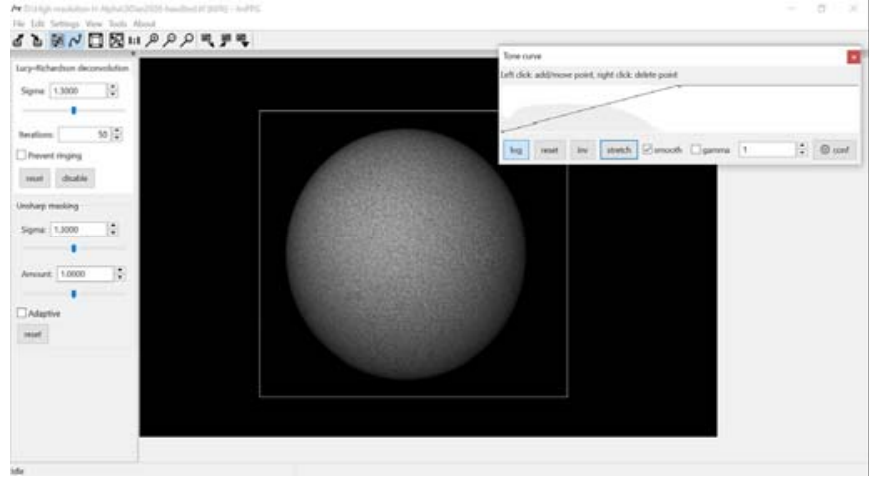

Figure 10. Stretching a tone curve in ImPPG

\subsubsection{Adobe Photoshop}

The sharpened image was then colored using the RGB tone curves in Adobe Photoshop. The color of the image was dependent on the layer of the Sun. Red-orangish colour was used for the upper chromosphere viewed using H-Alpha. Purplish-blue colour was used for lower chromosphere viewed using Calcium-K. Dark green colour was used for the photosphere viewed using white light. Then, an additional orientation step was used on some images to rotate the image so that the $\mathrm{x}$-axis of the image corresponds to the north and the $\mathrm{y}$-axis corresponds to the east. The orientation was done with respect to images from other solar observatories ${ }^{[2,3]}$. This was done in order to track solar features. 


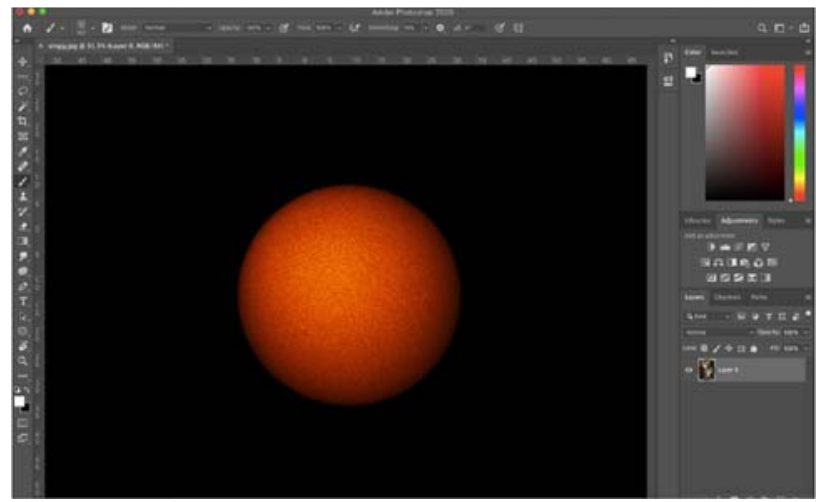

Figure 11. Colouring a H-Alpha image in Adobe Photoshop

\section{Results and discussion}

\subsection{Hydrogen-Alpha Images}

The images obtained with the Hydrogen-Alpha filter were monochrome, as a black and white camera was used. A reddish-orange pseudo-colour was added to the image (See Figure 12) as this colour will be produced by the filter if viewed through the eyepiece because we are looking at the upper chromosphere which emits a wavelength of $656.3 \mathrm{~nm}{ }^{[7]}$, which corresponds to this colour on the visible spectrum. Upon careful observation, an active region can be seen towards the south of the solar disk, which might develop into a sunspot. It has a streak like appearance and is brighter than the solar disk. However, this active region did not develop into a sunspot. Figure 13, shows a close-up view of the active region.

It should be noted that Figure 13 is not a high-resolution image and just a zoomed-in image. High-resolution imaging was not carried out in this research.

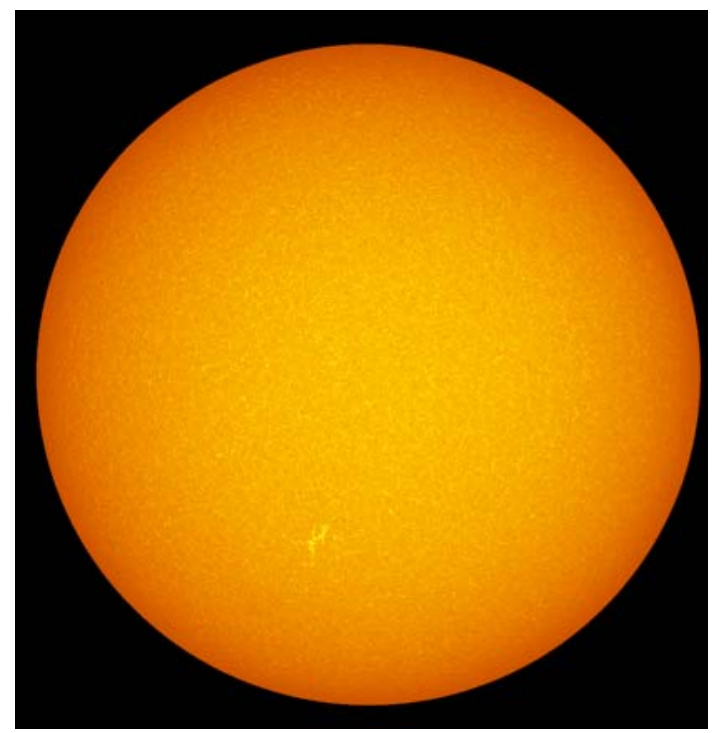

Figure 12. Upper Chromosphere seen via Hydrogen Alpha 


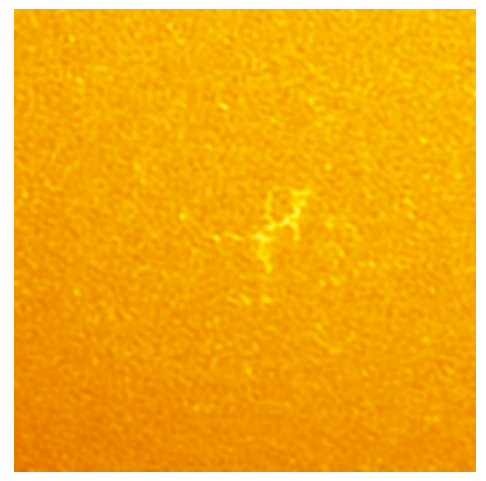

Figure 13. Active Region seen in H-Alpha

\subsection{Calcium-K Images}

Similar to H-Alpha, the Calcium-K images were monochrome. A purplish-blue pseudocolour was added (See Figure 14) as colour close to this will be produced if seen through the eyepiece because it is the lower chromosphere and it emits wavelength of $393.4 \mathrm{~nm}^{[8]}$ that corresponds to this colour on the visible spectrum. Figure 15 shows a close-up view of the solar limb. Again, it should be noted that it is not a high-resolution image. Unfortunately, no prominent solar activity is visible on the date of capture.

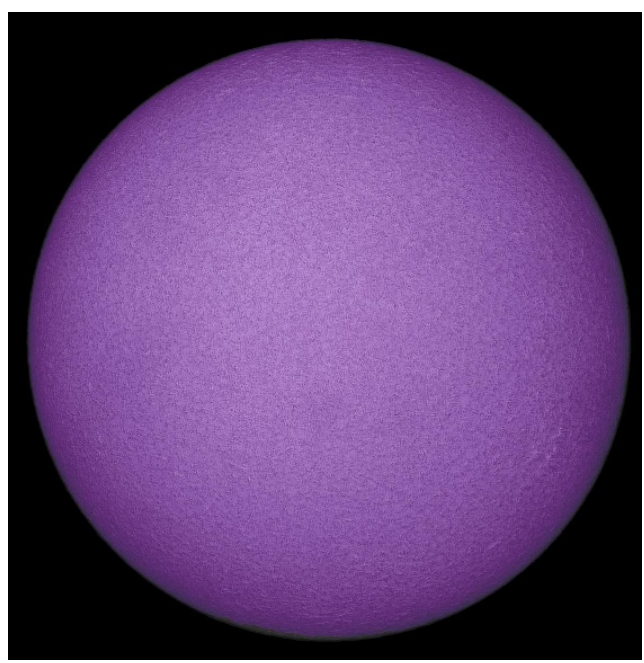

Figure 14. Lower Chromosphere seen via Calcium-K 


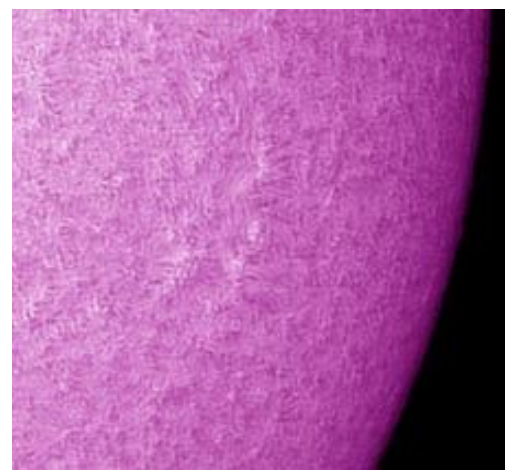

Figure 15. Close-up of Solar Limb in Calcium-K

\subsection{White Light Images}

Like the other two filters, the White Light images were monochrome as well. A dark green pseudo-colour was added to the image as colour close to this will be produced if seen through the eyepiece. This is the surface of the Sun, the photosphere, and we cannot see beyond this layer ${ }^{[9]}$. It can be seen that in Figure 16, no prominent solar activity of any kind is observed and the Sun appears inactive.

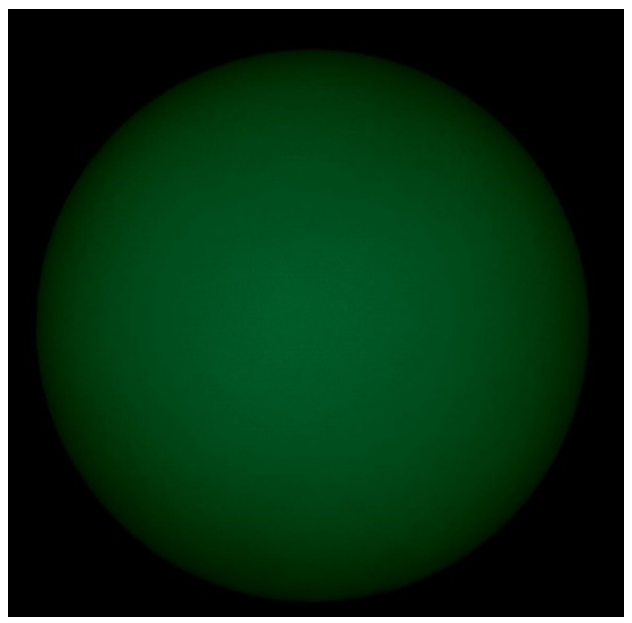

Figure 16. Photosphere seen through White Light

\section{Conclusion}

Not much solar activity can be seen on the Sun. The obtained images are very "plain" and "inactive". This is due to the fact that the Sun follows a 11-year activity cycle ${ }^{[5]}$. During the period of data collection, the Sun was at its solar minima, i.e., the period of minimum activity. 


\section{Future Works}

In the future, we are starting on high-resolution imaging to focus on certain parts of the Sun rather than observing the solar disk as a whole. We have already obtained some preliminary results for high-resolution imaging. Figure 17 shows a high-resolution image of a sunspot. The difference in contrast around the sunspot is clearly visible. Also, patterns formed by the magnetic field lines can be seen as well.

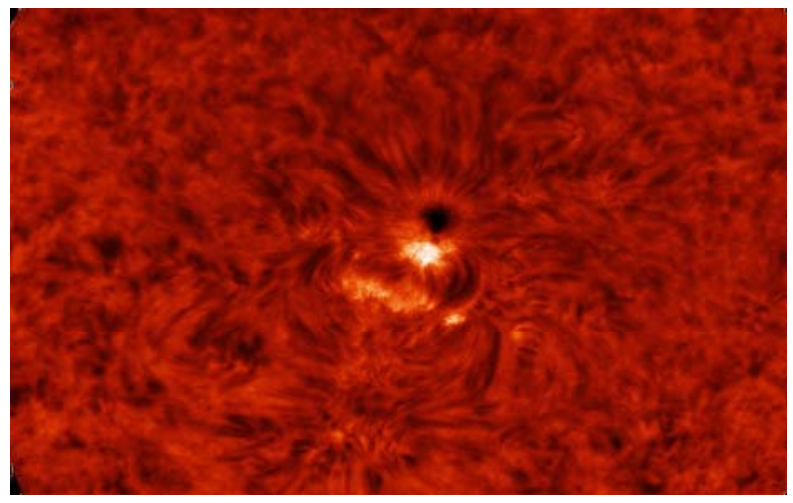

Figure 17. High-Resolution Image of a sunspot

Apart from high-resolution imaging, we might consider using the G-band filter as well to observe the Sun in a different wavelength.

\section{Safety Concerns}

- Handling of telescopes should be done with care as small shocks can cause the lenses to disorientate or damage the etalon.

- During data collection, it should be ensured that the filters are fitted on the telescope before any direct observation is made to ensure no harm is done to the eye.

- Proper sun protection gear - sunscreen, sunglasses, hats, et cetera - is used to prevent sunburns.

\section{Acknowledgements}

We would like to express our utmost gratitude to our research mentors, Ms. Ning Hwee Tiang and Mr. Alfred Tan, of National Junior College, for their continuous support and guidance throughout this research project. We would like to give special thanks to Mr. Alfred Tan, for letting us use his equipment and resources. We would also like to thank our schools, National Junior College and School of Science and Technology, for giving us the opportunity of this research project.

\section{References}

1. Sunspots and Solar Flares | NASA Space Place - NASA Science for Kids. (2019). Spaceplace.nasa.gov. Retrieved 25 December 2019, from https://spaceplace.nasa,govsolaractivity/en/ 
2. Addison, K. (2019). SDO | Solar Dynamics Observatory. Sdo.gsfc.nasa.gov. Retrieved 25 December 2019, from https://sdo.gsfc.nasa.gov/data/

3. Sun today - latest images of the Sun. (2019). Tesis.lebedev.ru. Retrieved 25 December 2019, from https://tesis.lebedev.ru/en/Sun pictures.html

4. D. Hathway, The Solar Cycle. Living Reviews In Solar Physics, 12, 1 (2015)

5. Solar experts predict the Sun's activity in Solar Cycle 25 to be below average, similar to Solar Cycle 24. (2019). Weather.gov. Retrieved 25 December 2019, from https://www.weather.gov/news/190504-Sun-activity-in-solar-cycle

6. Szczerek, F. (2020). ImPPG Processing Tutorial. Retrieved from https://greatattractor.github.io/ImPPG/tutorial/tutorial_en.html

7. Hydrogen Alpha Explained. (2020). Retrieved from http://www.astronomyknowhow.com/hydrogen-alpha.htm

8. 7 - Observing the Sun in Ca-K, Ca-H and Other Narrow Bandwidths $\mid$ AstronomyConnect. (2020). Retrieved from https://astronomyconnect.com/forums/articles/7-observing-the-Sunin-ca-k-ca-h-and-other-narrow-bandwidths.38/

9. The Photosphere - the "Surface" of the Sun | UCAR Center for Science Education. (2020). Retrieved from https://scied.ucar.edu/Sun-photosphere 